\title{
The role of CA-125, GLS and FASN in predicting cytoreduction for epithelial ovarian cancers
}

\author{
G. N. A. Winarno ${ }^{*}$ (D), Y. M. Hidayat ${ }^{1}$, S. Soetopo ${ }^{2}$, S. R. Krisnadi ${ }^{1}$, M. D. L. Tobing ${ }^{1}$ and S. Rauf ${ }^{3}$
}

\begin{abstract}
Objective: Cytoreduction has an important role in improving the survival rate of epithelial ovarian cancer (EOC) patients. This study aimed to assess the ability of preoperative serum CA125, FASN and GLS as predictors of cytoreductive surgery for epithelial ovarian cancer (EOC).

Results: The average values of serum CA-125, FASN, and GLS in the suboptimal cytoreduction group were higher than those in optimal cytoreduction group. The cut off point (COP) was $248.55(p=0.0001)$ with $73.2 \%$ sensitivity and $73.6 \%$ specificity for CA-125, 0.445 ( $p=0.017$ ) with $62.5 \%$ sensitivity and $60.4 \%$ specificity for FASN, and 22.895 $(p=0.0001)$ with $73.2 \%$ sensitivity and $75.5 \%$ specificity for GLS. The COP of CA-125 and GLS combined was 29.16 ( $p=0.0001$ ) with sensitivity $82.1 \%$ and specificity $73.6 \%$, while the COP of CA-125, GLS, and FASN combined was 0.83 ( $p=0.0001$ ) with $87.5 \%$ sensitivity and $73.6 \%$ specificity.
\end{abstract}

Keyword: Epithelial ovarian cancer, CA-125, FASN, GLS, Cytoreductive surgery

\section{Introduction}

With the recent advancements in science and technology, therapies for ovarian cancer is also continue to be developed. The management of ovarian cancer cases has developed in terms of surgical technology and chemotherapeutic regimens [1]. In evaluating the therapeutic response to treatment in ovarian cancer, clinicians continue to develop new drugs with targets related to cancer cell proliferation [1].

Despite many improvements in therapy for ovarian cancer patients, a study by Zhang Xi et al. study in China that compared the survival rate of EOC (epithelial ovarian cancer) between the September 1998-August 2007 and September 2007-August 2012 periods found only a meagre increase from 59.2 to $63 \%$ [2]. The latest report from the American Cancer Society states that the

\footnotetext{
*Correspondence: gatotnaw@yahoo.com

${ }^{1}$ Department of Obstetrics and Gynecology, Faculty of Medicine,

Padjadjaran University, Jl Pasteur No.38, Bandung, Indonesia

Full list of author information is available at the end of the article
}

survival rate of EOC for the period 2009-2015 was 47\% based on data on the incidence and trends of cancer in the United States [3].

Ovarian cancer is often referred to as the "Silent Killer" because most patients are already in an advanced stage upon diagnosis due to the lack of effective screening measures. The standard treatment for advanced stage EOC is cytoreductive surgery followed by adjuvant firstline platinum-taxane chemotherapy. Optimal cytoreduction is achieved with residual tumour of less than $1 \mathrm{~cm}$, while suboptimal cytoreduction is achieved with the residual tumour of more than $1 \mathrm{~cm} \mathrm{[4].}$

Several studies have shown a significant increase in survival rates in patients who have undergone optimal cytoreduction [5-7]. In a study conducted by Bacalbasa in 2015 with 99 patients with stage IIIC-IV ovarian cancer, the average survival duration of those who underwent optimal cytoreduction surgery was 72 weeks, while among patients who underwent suboptimal cytoreduction surgery, the duration was 51 weeks [8]. 
Optimal cytoreduction is the most convincing method of increasing survival time in patients with ovarian cancer [9]. However, additional exams such as computed tomography $(\mathrm{CT})$ and magnetic resonance imaging (MRI) are required to determine whether a patient is suitable for optimal cytoreduction. This has been the chief obstacle in providing optimal cytoreduction to ovarian cancer patients in Indonesia, where neither diagnostic modality is always available or affordable. Therefore, a serum biomarker that could predict the outcome of cytoreduction surgery in ovarian cancer could make the procedure more accessible in the absence of CT and MRI.

Fatty acid synthase (FASN) supports cancer cells to resist oxidative stress and limits the absorption of chemotherapy drugs $[10,11]$. There is a relationship between the stage of ovarian cancer and FASN levels; in one study, the level of FASN in end-stage cancer patients was 94.1\%, while in patients with stage I ovarian cancer, it was $12.5 \%$ [12].

Glutaminase (GLS) is an enzyme needed for the process of glutaminolysis which converts glutamine to glutamate.[13] Glutamine is an amino acid needed to support the growth of cancer cells and the formation of ATP.[14] In ovarian cancer with a high invasion rate, the rate of glutaminolysis will be higher than in ovarian cancer with a lower invasiveness.[15] This study assessed the use of preoperative serum CA-125, FASN, and GLS levels as predictors of cytoreduction in EOC, both individually and in combination.

\section{Material and methods}

The records of RSUP Dr. Hasan Sadikin Bandung were utilized to identify all patients with suspected EOC who underwent primary cytoreductive surgery from 2017 until 2019. This study was an observational analytic study with a cross-sectional design. The inclusion criteria in this study were ovarian cancer patients who underwent primary cytoreductive surgery, did not suffer from chronic diseases or other tumours, and were willing to participate in the study after completing informed consent forms. Patients who did not have epithelial-type ovarian cancer stage III-IV based on histopathology examination or with suboptimal histopathological preparations and blood serum were excluded in this study.

Preoperative serum FASN, GLS, and CA-125 levels, were independent variables while cytoreduction surgery, both optimal and suboptimal, was the dependent variable in this study. The cut-off point (COP) was determined using receiver operating characteristic (ROC) curve 23 analysis. The statistical analyses were performed with $\operatorname{SPSS}^{\mathrm{TM}}(24.0 .0)$.

The distributions of the variables were assed for normality with the Kolmogorov-Smirnov test.
Characteristics between the 2 groups were compared with the unpaired t-test (normally distributed) and the Mann-Whitney U test (non-normally distributed). Categorical data were compared with the Chi-squared test or, alternatively, Fisher's Exact test or and KolmogorovSmirnov test.

Blood samples were taken from the medial cubital vein (volume $1 \mathrm{~mL}$ ) before cytoreductive surgery. The blood samples of patients who had been confirmed by histopathological examination as EOC patients were examined using the enzyme-linked immunosorbent assay (ELISA) method at the Laboratory of Molecular Genetics of Padjajaran University.

Samples collected were left for $2 \mathrm{~h}$ at room temperature or overnight at $4{ }^{\circ} \mathrm{C}$ prior to centrifugation the next day, after which they were stored at $-20{ }^{\circ} \mathrm{C}$ or $-80{ }^{\circ} \mathrm{C}$ for later use. CA-125, FASN and GLS were assessed by ELISA kits (ADVIA Centaur CA125II, Siemens, United States; SEC470Hu, Cloud-Clone, United States, and SEJ026Hu, Cloud-Clone, United States, respectively) and prepared according to the manufacturer's instructions.

\section{Results}

\section{Characteristics of the sample}

One hundred nine patients were diagnosed with EOC during the study period. Most types of EOC were mucinous (34.9\%). 53 patients underwent suboptimal cytoreductive surgery, while 56 patients underwent optimal cytoreduction (Table 1).

\section{CA-125, FASN, and GLS serum levels}

The median values of serum of CA-125, FASN, and GLS in the suboptimal cytoreduction group were higher than those in the optimal cytoreduction group. For CA-125, the median value in the suboptimal cytoreduction group was $600 \mathrm{U} / \mathrm{mL}$, while in the optimal cytoreduction group the value was $120.30 \mathrm{U} / \mathrm{mL}(p=0.0001)$, FASN had a median value of $0.50 \mathrm{ng} / \mathrm{mL}$ in the suboptimal cytoreduction group and $0.37 \mathrm{ng} / \mathrm{mL}$ in the optimal cytoreductive surgery group $(p=0.006)$, and GLS had a median value of $25.25 \mathrm{ng} / \mathrm{mL}$ in the suboptimal cytoreduction group and $20.08 \mathrm{ng} / \mathrm{mL}$ in the optimal cytoreduction group $(p=0.0001)$.

\section{Biomarker serum as a predictor of cytoreductive surgery} The COP values for the different substances are shown in Table 2. The COP was 248.55 for CA-125, 0.445 for FASN, 22.895 for GLS, 0.69 for CA-125+FASN, 29.16 for CA-125+GLS, and 0.83 for CA-125+FASN+GLS. The formula for calculating the combined values involved multiplying the numerical value of the serum biomarker levels with the category values of the other serum biomarker levels, as described below. 
Table 1 Background characteristics of the study population

\begin{tabular}{|c|c|c|c|c|}
\hline \multirow[t]{3}{*}{ Variable } & \multirow[t]{3}{*}{$N=109$} & \multicolumn{2}{|l|}{ Group } & \multirow[t]{3}{*}{$P$ value } \\
\hline & & Suboptimal cytoreduction & Optimal cytoreduction & \\
\hline & & $\mathrm{N}=56$ & $\mathrm{~N}=53$ & \\
\hline Age (years) & & & & 0.269 \\
\hline Mean \pm Std & $47.48 \pm 11.347$ & $48.66 \pm 9.648$ & $46.24 \pm 12.880$ & \\
\hline Median & 47.00 & 47.00 & 47.00 & \\
\hline Range (min-max) & $17 / 00-75.00$ & $26.00-64.00$ & $17.00-75.00$ & \\
\hline Parity & & & & 0.261 \\
\hline 0 & $28(25.7 \%)$ & $16(28.6 \%)$ & $12(22.6 \%)$ & \\
\hline 1 & $16(14.7 \%)$ & $6(10.7 \%)$ & $10(18.9 \%)$ & \\
\hline 2 & $23(21.1 \%)$ & $15(26.8 \%)$ & $8(15.1 \%)$ & \\
\hline$>3$ & $42(38.5 \%)$ & $19(33.9 \%)$ & $23(43.4 \%)$ & \\
\hline IMT & & & & 0.353 \\
\hline Mean \pm Std & $21.30 \pm 3.907$ & $20.96 \pm 3.675$ & $21.66 \pm 4.143$ & \\
\hline Median & 21.30 & 21.25 & 21.40 & \\
\hline Ascites & & & & 0.089 \\
\hline Mean \pm Std & $1652.56 \pm 3664.119$ & $1703.75 \pm 3393.296$ & $1598.49 \pm 3962.289$ & \\
\hline Median & 300.00 & 400.00 & 300.00 & \\
\hline Stage & & & & 0.0001 \\
\hline$\|$ & $42(38.5 \%)$ & $8(14.3 \%)$ & $34(64.2 \%)$ & \\
\hline III & $57(52.3 \%)$ & $38(67.9 \%)$ & $19(35.8 \%)$ & \\
\hline IV & $10(9.2 \%)$ & 10 (17.9\%) & $0(0.0 \%)$ & \\
\hline Histopathology & & & & 0.559 \\
\hline Serous & $27(24.8 \%)$ & $18(32.1 \%)$ & $9(17.0 \%)$ & \\
\hline Mucinous & 38 (34.9\%) & $12(21.4 \%)$ & $26(49.1 \%)$ & \\
\hline Endometrioid & $21(19.3 \%)$ & 14 (25.0\%) & $7(13.2 \%)$ & \\
\hline Clear cell & $16(14.7 \%)$ & $8(14.3 \%)$ & $8(15.1 \%)$ & \\
\hline Others & 7 (6.4\%) & $4(7.1 \%)$ & $3(5.7 \%)$ & \\
\hline
\end{tabular}

For the CA-125+FASN and CA-125+ GLS combinations, the COP value was calculated using CA-125 as the category variable $(1 ; \leq 248.55,2>248.55)(p=0.0001)$. The formula for the combined value of CA-125+FASN + GLS used CA-125 $(1 ; \leq 248.55,2>248.55)$ and GLS $(1 ; \leq 22.895$, $2>22.895)$ as the category variables $(p=0.0001)$.

The ROC curves for each of the biomarkers are presented in Fig. 1. The AUC of the ROC curve (Fig. 1) for CA-125 was $76,7 \%(p=0.0001)$, for FASN was $65.3 \%(p=0.006)$, for GLS was $74.1 \%(p=0.0001)$, for CA-125+FASN was $76.9 \%$, for CA-125+GLS was $85.4 \%$ and for CA-125+FASN + GLS was $87.7 \%$. The highest AUC was obtained for the combined CA-125+FASN + GLS (Fig. 1).

\section{Discussion}

Several methods can be used to predict the results of cytoreductive operations, from the history-taking, physical examination, supporting examinations such as ultrasound, CT, MRI, PET, and laboratory [16-18].
Some studies found that CA-125 is not a sufficiently strong predictor for cytoreduction surgery $[10,19,20]$. In this study, the researchers aimed to assess other biomarkers, namely FASN and GLS, separately and in different combinations.

In his study, Memarzadeh et al. determined that CA-125 was still a very weak predictor for cytoreduction surgery because of its low sensitivity $(58 \%)$ and a spesificity (54\%) [21]. The cut-off point, $500 \mathrm{U} / \mathrm{mL}$, used by Obeidat Basil, et al. was obtained from Chi's study with stage III ovarian cancer patients as the samples [22], yielding a sensitivity of $72 \%$ and specificity of $73 \%$ for predicting suboptimal cytoreduction surgery by CA-125 [19]. However, in this study, the cut-off point value for CA-125 was $248.55 \mathrm{U} / \mathrm{mL}$, with a sensitivity of $73.2 \%$, spesificity of $73.6 \%$, and accuracy of $73.3 \%(p=0.0001)$.

CA-125, commonly referred to as MUC16, is a glycoprotein that protects the cell layer from external threats and supports the epithelial-mesenchymal transition, the essential process of metastasis in ovarian cancer [23, 24]. 


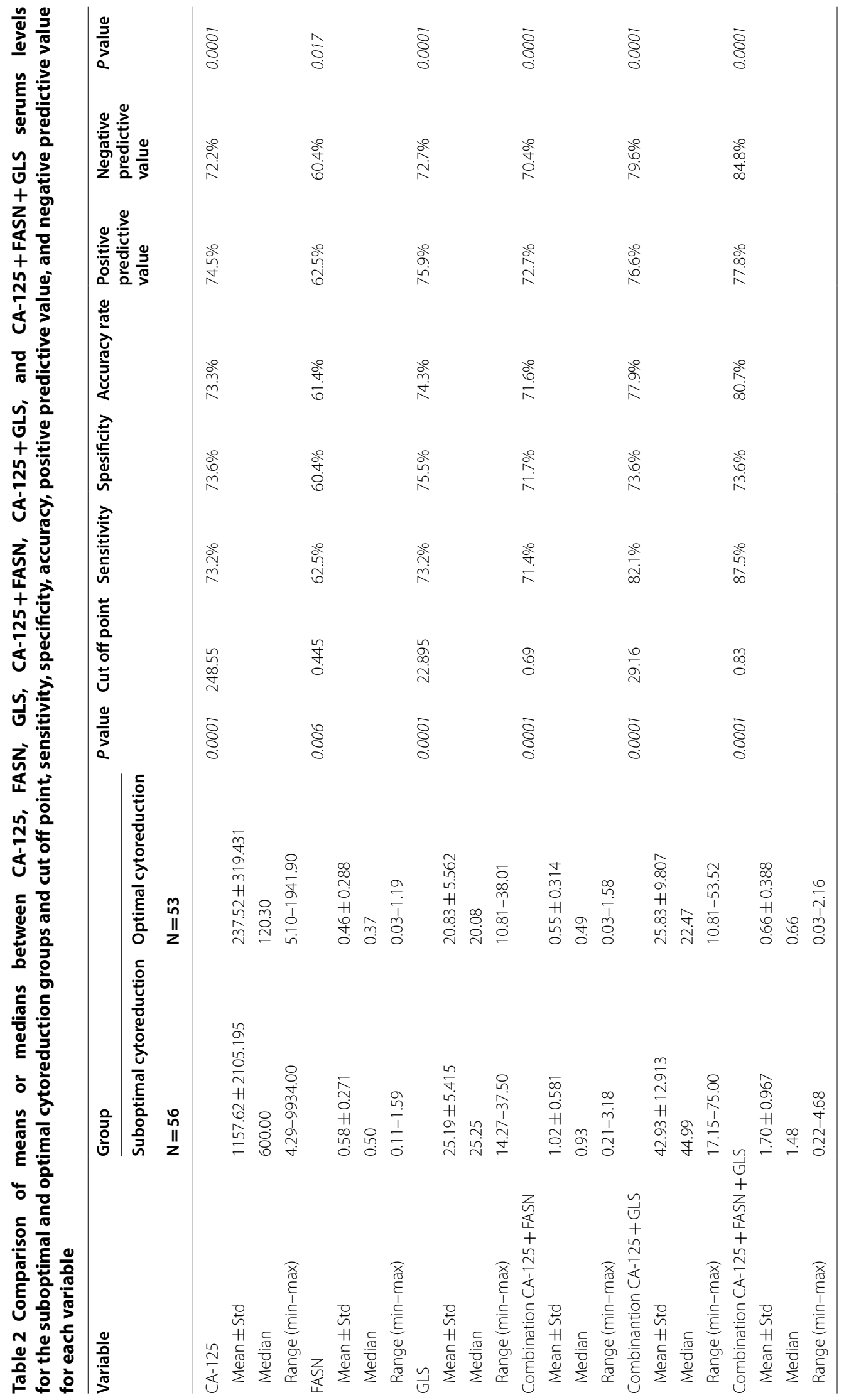



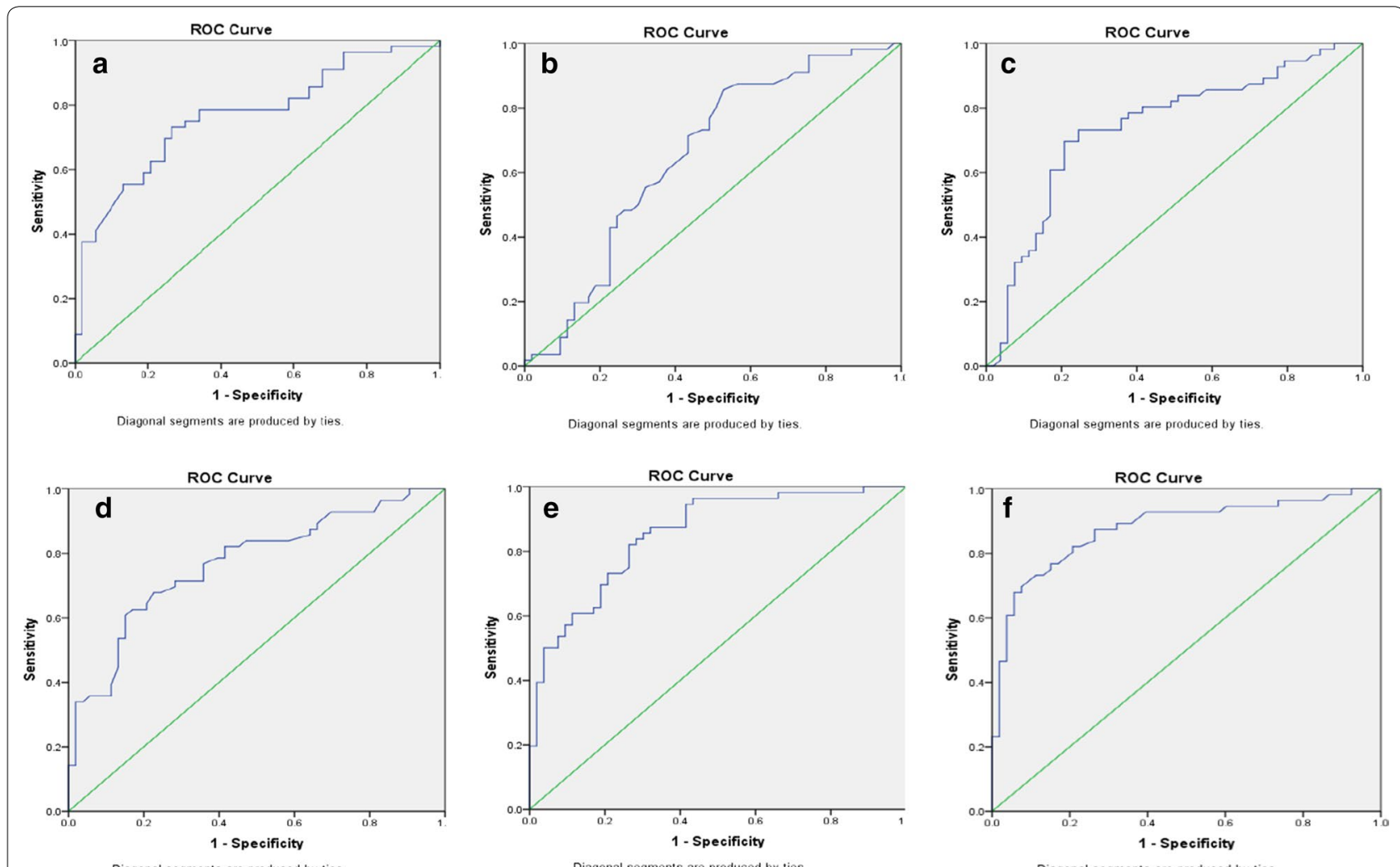

Diagonal segments are produced by ties

Diagonal segments are produced by ties

Diagonal segments are produced by ties

Fig. 1 ROC curves. a ROC curve for the value of CA-125 in predicting cytoreduction. The AUC was 76.7\% (Cl 67.8-85.6\%, $p=0.000)$, implying that CA-125 can predict cytoreduction correctly in 84 patients out of a total of 109 patients. b ROC curve for the value of FASN in predicting cytoreduction. The AUC was $65.3 \%$ (CI 54.8-75.8\%, $p=0.006$ ), implying that FASN can predict cytoreduction correctly in 71 patients out of a total of 109 patients. c ROC curve for the value of GLS in predicting cytoreduction. The AUC was $74.1 \%(\mathrm{Cl} 64.6-83.7 \%, p=0.0000)$, implying that GLS can predict cytoreduction correctly in 81 patients out of a total of 109 patients. d ROC curve for the value of FASN and CA-125 in predicting cytoreduction. The AUC was $76.9 \%$ (Cl 68.1-85.8\%, $p=0.0000$ ), implying that FASN and CA-125 can predict cytoreduction correctly in 84 patients out of a total of 109 patients. e ROC curve for the value of GLS and CA-125 in predicting cytoreduction. The AUC was 85.4\% (Cl 78.4-92.3\%, $p=0.0000$ ), implying that GLS and CA-125 can predict cytoreduction correctly in 91 patients out of a total of 109 patients. $\mathbf{f}$ ROC curve for the value CA-125, FASN and GLS in predicting ytoreduction. The AUC was 87.7\% (Cl 81-94.4\%, $p=0.0000)$, implying that CA-125, FASN and GLS can predict cytoreduction correctly in 96 patients out of a total of 109 patients

MUC16 has been identified as the most highlt expressed antigen in ovarian cancer [10].

Metabolic changes occur to supply proliferative cancer cells. These cells require the formation of adenosine triphosphate (ATP) in the fulfillment of their energy needs, which itself requires changes in the metabolic pathways of carbohydrates, proteins, lipids, and nucleic acids [25].

Lipids are molecules consisting of fatty acids and can be obtained exogenously and endogenously (de novo lipogenesis) [26]. These fatty acids are used to produce ATP in the proliferation of cancer cells, and the fatty acids produced by de novo lipogenesis protect cancer cells from free radicals and chemotherapy by forming the structure of cancer cell membranes [27]. These fatty acids are formed from the conversion of 12 of malonyl-CoA into palmitate with the help of FASN [28].
FASN has been shown to induce epithelial-mesenchymal transitions, thereby increasing the chances of ovarian cancer metastasis to the peritoneal cavity [29]. Therefore, tumours that have an aggressive phenotype will show increased FASN expression [30]. These results provided the basis for the researchers to pursue FASN as an alternative predictor to CA-125 for cytoreduction. However, we found that FASN was a weaker predictor than CA-125, having lower sensitivity. The combined FASN + CA-125 also did not seem to give better results than CA-125 itself, with a sensitivity of $71.4 \%$ and a specificity of $71.7 \%(p=0.0001)$.

The primary source of nutrition that provides bioenergy to cancer cells for proliferation is glutamine [31]. The characteristic metabolic change in cancer cells is an increase in glutaminolysis. Glutamine is consumed by tumour cells ten times more than other amino acids 
because of its function in the proliferation and viability of most cancer cells [32]. Compared to that in healthy cells, the conversion of glutamine to lactate in cancer cells is incrased.[31] Because of its essential function in cancer cell metabolism, if glutamine deficiency occurs, cell growth inhibition can even induce apoptosis [31]. Yang et al. found that the level of cancer cell dependence on glutamine was highly correlated with the rate of cancer invasion [31]. Glutamine is metabolized through the Krebs cycle and influenced by 2 enzymes, namely GLS and glutamate dehydrogenase (GDHS) [13].

As a predictor of cytoreduction, GLS yielded a sensitivity of $73.2 \%$ and a specificity of $75.5 \%$ with a cut-off point of $22.895(p=0.0001)$. The combined GLS + CA-125 yielded better results than the individual biomarkers, with $82.1 \%$ sensitivity and $73.6 \%$ specificity. To the best of our knowledge, this is the first study to use FASN and GLS as predictors for cytoreduction surgery in EOC. Our study determined that GLS possesses a sensitivity and specificity almost equivalent to those of CA- 125 .

CA-125 had an accuracy of 73.3\%, while GLS had an accuracy of $74.3 \%$. Different combinations of biomarkers had increased sensitivity, specificity, and accuracy values in predicting cytoreduction. CA-125+FASN + GLS had a higher accuracy $(80.7 \%)$ than CA-125+GLS $(77.9 \%)$ or CA-125+FASN (71.6\%). Therefore, the clinicians could use this combination as an alternative to predict cytoreductive surgery in EOC.

\section{Conclusion}

In summary, the individual roles of CA125, FASN and GLS levels in predicting suboptimal cytoreductive surgery for patients with EOC is questionable. However, the combination of CA-125 and GLS or CA-125, FASN and GLS can increase the sensitivity, specificity, and accuracy in predicting suboptimal cytoreductive surgery. The combined score is expected to help doctors to provide better therapy than before.

\section{Limitations}

One of the limitations of this study was the use of stages II, III, and IV in epithelial ovarian cancer, as the staging of the cancer affected the tpe of cytoreductive surgery in this study (Table 1.). CA-125 is thought to be unable to predict cytoreduction because of its low value in mucinous-type ovarian cancer, but it is not known how other serum biomarker values such as FASN and GLS perform for this kind of cancer. The researchers were unable to assess the serum biomarkers in each category of epithelial ovarian cancer due to the lack of samples in each category.

\section{Abbreviations}

CA-125: Cancer antigen 125; EOC: Ephitelial ovarian cancer; FASN: Fatty acid synthase; GLS: Glutaminase; ROC: Receiver operating characteristic; AUC: Area under the curve.

\section{Acknowledgements}

"I would like to thank the Department of Obstetrics and Gynecology, RSUP Dr. Hasan Sadikin, Universitas Padjajaran Bandung, and my PhD advisors during the study".

\section{Authors' contributions}

GNAW: Protocol/project development, Data collection or management, Data analysis, Statistical analysis, Literature Search, Manuscript writing/editing. YMH: Protocol/project development, Data collection or management, Data analysis, Statistical analysis. SS: Protocol/project development, Data collection or management, Statistical analysis. SRK: Protocol/project development, Literature Search, Manuscript writing/editing. MDLT: Protocol/project development, Literature Search. SR: Protocol/project development, Statistical analysis. All authors read and approved the final manuscript.

\section{Funding}

Author declares no funding resources.

\section{Availability of data and materials}

The datasets used and/or analysed during the current study are available from the corresponding author on reasonable request.

\section{Ethical approval and consent to participate}

The study protocol was approved by Faculty of Medicine Universitas Padjajaran, Ethics Committee Review Board, refference number 0518010184 and all studsy participants gave informed consent, patient consents to participate was written. All authors hereby declare that all patients have been examined in accordance with the ethical standards laid down in the 1964 Declaration of Helsinki.

\section{Consent for publication}

All authors read and approved the final manuscript.

\section{Competing interest}

The authors declare that they have no conflicts of interest.

\section{Author details \\ ${ }^{1}$ Department of Obstetrics and Gynecology, Faculty of Medicine, Padjadjaran University, Jl Pasteur No.38, Bandung, Indonesia. ${ }^{2}$ Department of Radiol- ogy, Faculty of Medicine, Padjadjaran University, Jl Pasteur No.38, Bandung, Indonesia. ${ }^{3}$ Department of Obstetrics and Gynecology, Faculty of Medicine, Hasanuddin University, Jl. Perintis Kemerdekaan KM. 10, Makasar, Indonesia.}

Received: 7 June 2020 Accepted: 15 July 2020

Published online: 22 July 2020

\section{References}

1. Cortez AJ, Tudrej P, Kujawa KA, Lisowska KM. Advances in ovarian cancer therapy. Cancer Chemother Pharmacol. 2018;81 (1):17-38.

2. Xi Z, Qing Z, Kun S, Baoxia C, Jie J, Youzhong Z, et al. Five-year survival rate of postoperative ovarian cancer patients: a 15-year retrospective study. 现代妇产科进展. 2017;12:26.

3. Noone A, Howlader N, Krapcho M, Miller D, Brest A, Yu M, et al. SEER Cancer Statistics Review, 1975-2015. Bethesda: National Cancer Institute; 2018.

4. Morgan RJ, Armstrong DK, Alvarez RD, Bakkum-Gamez JN, Behbakht K, Chen L-M, et al. NCCN Clinical Practice Guidelines in Oncology (NCCN Guidelines ${ }^{\circledR}$ ). Ovarian cancer, including fallopian tube cancer and primary peritoneal cancer: NCCN.org 2015. 2015. https://www.nccn.org/profe ssionals/physician_gls/pdf/ovarian.pdf. Accessed 22 Juni 2015

5. Bristow RE, Montz FJ, Lagasse LD, Leuchter RS, Karlan BY. Survival impact of surgical cytoreduction in stage IV epithelial ovarian cancer. Gynecol Oncol. 1999;72(3):278-87. 
6. Shih KK, Chi DS. Maximal cytoreductive effort in epithelial ovarian cancer surgery. J Gynecol Oncol. 2010;21 (2):75-80.

7. Colombo P-E, Mourregot A, Fabbro M, Gutowski M, Saint-Aubert B, Quenet $F$, et al. Aggressive surgical strategies in advanced ovarian cancer: a monocentric study of 203 stage IIIC and IV patients. Eur J Surg Oncol. 2009;35(2):135-43.

8. Bacalbasa N, Balescu I, Dima S, Herlea V, David L, Brasoveanu V, et al. Initial incomplete surgery modifies prognosis in advanced ovarian cancer regardless of subsequent management. Anticancer Res. 2015;35(4):2315-20.

9. Bristow RE, Tomacruz RS, Armstrong DK, Trimble EL, Montz F. Survival effect of maximal cytoreductive surgery for advanced ovarian carcinoma during the platinum era: a meta-analysis. J Clin Oncol. 2002;20(5):1248-59.

10. Haridas D, Ponnusamy MP, Chugh S, Lakshmanan I, Seshacharyulu P, Batra SK. MUC16: molecular analysis and its functional implications in benign and malignant conditions. FASEB J. 2014;28(10):4183-99.

11. Kang S, Kim TJ, Nam BH, Seo SS, Kim BG, Bae DS, et al. Preoperative serum CA-125 levels and risk of suboptimal cytoreduction in ovarian cancer: a meta-analysis. J Surg Oncol. 2010;101(1):13-7.

12. Cai Y, Wang J, Zhang $L, W u ~ D, Y u ~ D$, Tian $X$, et al. Expressions of fatty acid synthase and HER2 are correlated with poor prognosis of ovarian cancer. Med Oncol. 2015;32(1):391.

13. Rahman M, Hasan MR. Cancer metabolism and drug resistance. Metabolites. 2015;5(4):571-600.

14. Daye D, Wellen KE. Metabolic reprogramming in cancer: unraveling the role of glutamine in tumorigenesis. Semin Cell Dev Biol. 2012;23(4):362-9.

15. Erickson JW, Cerione RA. Glutaminase: a hot spot for regulation of cancer cell metabolism? Oncotarget. 2010;1(8):734.

16. Testa AC, Ludovisi M, Mascilini F, Di Legge A, Malaggese M, Fagotti A, et al. Ultrasound evaluation of intra-abdominal sites of disease to predict likelihood of suboptimal cytoreduction in advanced ovarian cancer: a prospective study. Ultrasound Obstet Gynecol. 2012;39(1):99-105.

17. Qayyum A, Coakley FV, Westphalen AC, Hricak H, Okuno WT, Powell B. Role of CT and MR imaging in predicting optimal cytoreduction of newly diagnosed primary epithelial ovarian cancer. Gynecol Oncol. 2005;96(2):301-6.

18. Bristow RE, del Carmen MG, Pannu HK, Cohade C, Zahurak ML, Fishman EK, et al. Clinically occult recurrent ovarian cancer: patient selection for secondary cytoreductive surgery using combined PET/CT. Gynecol Oncol. 2003;90(3):519-28.

19. Obeidat B, Latimer J, Crawford R. Can optimal primary cytoreduction be predicted in advanced stage epithelial ovarian cancer? Role of preoperative serum CA-125 level. Gynecol Obst Invest. 2004;57(3):153-6.
20. Barlow T, Przybylski M, Schilder J, Moore D, Look K. The utility of presurgical CA125 to predict optimal tumor cytoreduction of epithelial ovarian cancer. Int J Gynecol Cancer. 2006;16(2):496-500.

21. Memarzadeh S, Lee S, Berek J, Farias-Eisner R. CA125 levels are a weak predictor of optimal cytoreductive surgery in patients with advanced epithelial ovarian cancer. Int J Gynecol Cancer. 2003;13(2):120-4.

22. Chi DS, Venkatraman ES, Masson V, Hoskins WJ. The ability of preoperative serum CA-125 to predict optimal primary tumor cytoreduction in stage III epithelial ovarian carcinoma. Gynecol oncol. 2000;77(2):227-31.

23. Bottoni P, Scatena R. The role of CA 125 as tumor marker: biochemical and clinical aspects. Adv Exp Med Biol. 2015;867:229-44.

24. Comamala M, Pinard M, Theriault C, Matte I, Albert A, Boivin M, et al. Downregulation of cell surface CA125/MUC16 induces epithelial-to-mesenchymal transition and restores EGFR signalling in NIH: OVCAR3 ovarian carcinoma cells. Br J Cancer. 2011;104(6):989-99.

25. Cairns RA, Harris IS, MakTW. Regulation of cancer cell metabolism. Nat Rev Cancer. 2011;11(2):85-95.

26. Menendez JA, Lupu R. Fatty acid synthase and the lipogenic phenotype in cancer pathogenesis. Nat Rev Cancer. 2007;7(10):763-77.

27. Rysman E, Brusselmans K, Scheys K, Timmermans L, Derua R, Munck S, et al. De novo lipogenesis protects cancer cells from free radicals and chemotherapeutics by promoting membrane lipid saturation. Cancer res. 2010;70(20):8117-266.

28. Zhang F, Du G. Dysregulated lipid metabolism in cancer. World J Biol Chem. 2012;3(8):167-74.

29. Saygili U, Guclu S, Uslu T, Erten O, Demir N, Onvural A. Can serum CA-125 levels predict the optimal primary cytoreduction in patients with advanced ovarian carcinoma? Gynecol Oncol. 2002;86(1):57-61.

30. Ameer F, Scandiuzzi L, Hasnain S, Kalbacher H, Zaidi N. De novo lipogenesis in health and disease. Metabolism. 2014;63(7):895-902.

31. Yuan $L$, Sheng $X$, Willson AK, Roque DR, Stine JE, Guo H, et al. Glutamine promotes ovarian cancer cell proliferation through the mTOR/S6 pathway. Endocr Relat Cancer. 2015;22(4):577-91.

32. Zhang Y, Yang J-M. Altered energy metabolism in cancer: a unique opportunity for therapeutic intervention. Cancer Biol Ther. 2013;14(2):81-9.

\section{Publisher's Note}

Springer Nature remains neutral with regard to jurisdictional claims in published maps and institutional affiliations.

Ready to submit your research? Choose BMC and benefit from:

- fast, convenient online submission

- thorough peer review by experienced researchers in your field

- rapid publication on acceptance

- support for research data, including large and complex data types

- gold Open Access which fosters wider collaboration and increased citations

- maximum visibility for your research: over $100 \mathrm{M}$ website views per year

At BMC, research is always in progress.

Learn more biomedcentral.com/submissions 